\title{
Analysis on the Co-authoring in the Field of Management in China: Based on Social Network Analysis
}

\author{
https://doi.org/10.3991/ijet.v12i06.7091 \\ Chuanyi Wang \\ Wuhan University, HuBei Wuhan, China \\ Zhe Cheng* \\ Wuhan University, HuBei Wuhan, China \\ 00030854 @whu .edu.cn \\ Zhiwei Huang \\ Wuhan University, HuBei Wuhan, China
}

\begin{abstract}
Using bibliographic data extracted from CNKI database, social network analysis is used to generate and analyze the network of co-authors of China in the field of management. This article suggests that: the density of the network is low, which means the collaboration between authors in China is not tight; the relations between the degree centrality and research output are weak. The author who published more papers may not have more co-authors. Through the lens of betweenness centrality, several authors in key positions of network are always dominating the academic information exchange and the small groups of authors have changed from 2006 to 2015 . The result of core-periphery analysis reflects that only a very small proportion of scholars are in the core of the network while most are relatively independent. The similarity of working experience, academic authority and geographical closeness are helpful to form and enhance the collaboration network.
\end{abstract}

Keywords - social network analysis, co-author, management

\section{Introduction}

With the development of science and technology, the integration, differentiation and cross-fertilization of disciplines are increasingly important. Therefore, scholars with different backgrounds start seeking collaborations for better development. Research collaboration becomes a vital force to promote the development of disciplines.

A co-authorship network is a social network consisting of a collection of researchers each of whom is connected to one or more other researchers if they have coauthored one or more papers and such a network can be represented as a set of nodes (or vertices) denoting co-authors joined by edges (or links) denoting research acquaintance. ${ }^{1}$ 
This study employed Social Network Analysis to explore the characteristics of coauthorship network in the field of management in China so as to explore the trend of research collaboration between scholars in past 10 years.

\section{Research Methodology and Data}

\subsection{Methodology}

Social Network Analysis is used to gain an understanding of the nodes (co-authors) and relationships (those who wrote a paper together) in the co-authorship network. In this study, we will analyze the density, centrality, core groups and small groups within the network.

\subsection{Data}

The data is collected from the database of China National Knowledge Infrastructure $(\mathrm{CNKI})$, and 5 top Chinese journals in the field management were selected including Journal of Management Sciences, Journal of Industrial Engineering and Management, Chinese Journal of Management Science, Chinese Public Administration and Chinese Journal of Hospital Administration. The retrieval time is from 2006 to 2015. There are 8830 papers after deleting the book reviews, editorial material and meeting abstract.

\section{An Overall Analysis of Co-authors}

\subsection{Trend of Co-authorship Rate}

At first, we focus on the co-authored publications. This was achieved by Excel to extract a list of co-authors. Another software called Bibexcel was then used to convert this list of authors into network file to the format which is readable by Ucinet.

Through the preliminary dealing with data, there are 5,808 collaborative papers, accounting for $65.76 \%$ of the total, in 8830 samples. All the literatures involved 10,653 authors, including 6459 co-authors, accounting for $60.63 \%$ of the total number of authors.

Table 1. Proportion of papers with more than 1 author

\begin{tabular}{|c|c|c|}
\hline Numbers of author & Numbers of articles & Proportion \\
\hline 2 & 3055 & $34.60 \%$ \\
\hline 3 & 2026 & $22.94 \%$ \\
\hline 4 & 522 & $5.91 \%$ \\
\hline 5 & 132 & $1.49 \%$ \\
\hline$>=6$ & 73 & $0.82 \%$ \\
\hline
\end{tabular}


From Fig.1, we can clearly see that the co-authorship rates of the five journals are in a gradual upward trend. And the percentage of the co-authors is about $60 \%$ $70 \%$, proving that the co-authorship in the research is a normal state. From the results, we can deduce that the collaboration between scholars will increase in the future. And there will be more interdisciplinary, cross-cutting collaborative research.

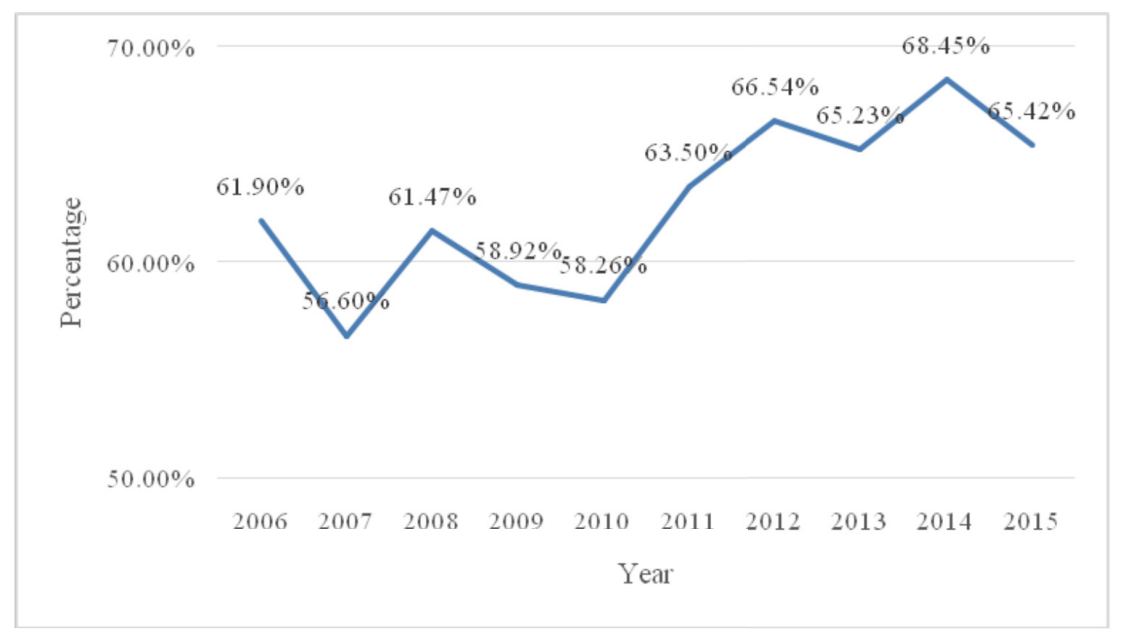

Fig. 1. Co-authorship Rate

\subsection{Identification of core authors}

The network constructed by massive authors would be extremely complex and not helpful for the further analysis in the subnets. In order to highlight the collaborationrelationship intuitively, the number of co-authors needs to be limited to narrow the scope of analysis. According to Price's law (Equation 1), we select the productive authors with 8 or more papers to construct co-author matrix. Top ten core authors are shown in Table2.

$$
\mathrm{M}=0.749\left(\mathrm{X}_{\max }\right)^{0.5}
$$

$\mathrm{M}$ is the lowest value of the core author; $\mathrm{X}_{\max }$ is the number of papers published by the author with the most productive.

Table 2. Top Ten Core Authors

\begin{tabular}{|c|l|c|c|l|c|}
\hline \multicolumn{1}{|c|}{ Author } & Papers & & \multicolumn{1}{c|}{ Author } & Papers \\
\hline 1 & Xia Shu-zhang & 132 & 6 & Gao Xiao-ping & 36 \\
\hline 2 & Ji Zhe1 & 108 & 7 & Liu Jie & 36 \\
\hline 3 & Ji Zhe2 & 60 & 8 & Da Qing-li & 35 \\
\hline 4 & Liu Si-feng & 49 & 9 & Liu Xing & 32 \\
\hline 5 & Tang Xiao-wo & 43 & 10 & He Jian-min & 31 \\
\hline
\end{tabular}

Note: Jizhe1 and Jizhe2 are homonyms of Chinese names. 


\section{$4 \quad$ Visualization of Scientific Collaboration Network}

We divided the ten years into two phases and generate a picture by net-draw. The result of visualization is shown in Fig.2.
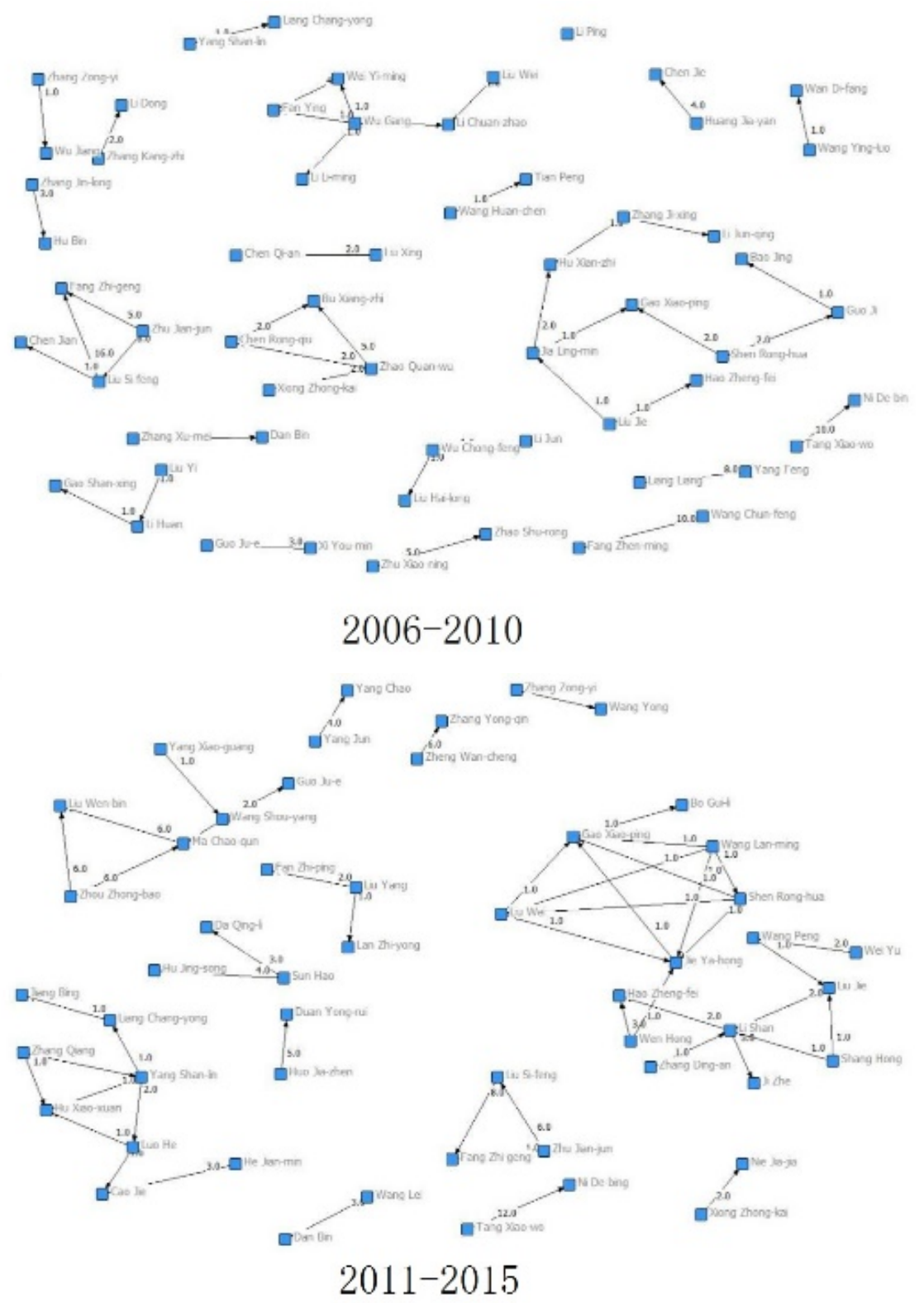

Fig. 2. Scientific Collaboration Network of Core Authors (2006-2010,2011-2015) 
Through the comparative analysis of the two periods, we find that:

The scientific collaboration network continues to expand, and new groups emerge. Taking the network of Gao Xiao-ping for example, though the collaboration network is still small, the number of co-authorship papers had obviously expanded in the last five years. Overall the co-authorship rate in the former five years was $62.8 \%$, the latter five-year co-authorship rate was $68.87 \%$, which has significant increased. Taking Tang Xiao-wo and Ni De-bing for example, the numbers of co-authorships in the former five years are 10, and in the latter five years have risen to 12 .

Besides, the scientific collaboration networks are changing. Some scientists have withdrawn from the network, while others have joined in. In addition, some authors have changed their network. Liu Wei, for example, he was in the team of Wu Gang at the first five years, then he joined the team of Gao Xiao-ping at the second five years.

\section{$5 \quad$ Scientific Collaboration Network Analysis}

\subsection{Network Density Analysis}

Density refers to the "connections" between core authors. If every node is directly connected to every other node, we have a complete graph. The density of a graph is defined as the number of links divided by the number of vertices in a complete graph with the same number of nodes. ${ }^{2}$ For an undirected graph $G$ with $N$ nodes, the density $D$ is defined as Equation 2:

$$
D=\frac{2^{*}(\# L(G))}{N(N-1)}
$$

And the research has proven that the density of the network affects the dissemination of knowledge and information. ${ }^{3}$ The greater the density, the more conducive to the sharing and dissemination of knowledge.

Table 3. Network Density Analysis

\begin{tabular}{|c|c|}
\hline Period & Density \\
\hline $2006-2010$ & 0.0085 \\
\hline $2011-2015$ & 0.0111 \\
\hline $2006-2015$ & 0.0084 \\
\hline
\end{tabular}

According to the results of network density analysis, the network density of the coauthors is 0.0084 in 10 years. It proves that the collaboration among the core authors is not tight. At the same time, it also shows that in the field of management, there is still much space for scientific collaboration. However, in the latter five year, the density has improved, which means the collaboration in the field is going up. 


\subsection{Network Centrality Analysis}

Centralization is used to describe the status and importance of individuals or organizations in their social networks and the most important centrality measures including degree centrality, closeness centrality and betweenness centrality. ${ }^{4}$

Degree Centrality: Degree centrality is simply the degree of a vertex, which can be measured by the number of nodes directly connected to it. ${ }^{5}$

Table 4. Centrality analysis

\begin{tabular}{|l|c|c|}
\hline \multicolumn{1}{|c|}{ Name } & Degree & Nrm Degree \\
\hline Da Qing-li & 19 & 0.833 \\
\hline Zhu Jian-jun & 18 & 0.789 \\
\hline Liu Si-feng & 12 & 0.526 \\
\hline Gao Xiaoping & 11 & 0.482 \\
\hline Wang Wen-bin & 11 & 0.482 \\
\hline$\ldots \ldots$ & $\ldots \ldots$ & $\ldots \ldots$ \\
\hline Wang Di-fang & 1 & 0.044 \\
\hline Huang He & 1 & 0.044 \\
\hline
\end{tabular}

In Table4, the third column (Degree) is the absolute degree of centrality, the fourth column (NrmDegree) is the relative degree of centrality. We can conclude that the highest degree is Da Qing-li, the absolute degree is 19 points. That means, Da QingLi once published papers in the five top journals with 19 authors. The minimum value of the absolute point centrality is 1 , which indicates the author collaborated with only one scholar.

Betweenness centrality: Betweenness is a measure which measures the extent to which a particular node lies between the various other nodes of the network. ${ }^{6}$ Betweenness centrality is defined as the ratio of the number of shortest paths (between all pairs of nodes) that pass through a given node divided by the total number of shortest paths. The normalized betweenness centralitybi of node $i$ is given as Equation 2:

$$
\sum_{i=k^{\wedge} i \neq j \neq k} \frac{g_{j i k}}{g_{j k}} / \frac{(n-1)(n-2)}{2}
$$

where $n$ is the number of nodes, $g_{j k}$ is the number of shortest paths from node $j$ to node $k$, and $g_{j i k}$ is the number of shortest paths from node $j$ to node $k$ that pass $b$ through node $i .^{7}$ 
Table 5. The Betweeness centrality of the top ten authors

\begin{tabular}{|l|c|c|l|c|c|}
\hline \multicolumn{1}{|c|}{ Name } & Betweenness & $\begin{array}{c}\text { Betweeness } \\
\text { eentrality }\end{array}$ & \multicolumn{1}{|c|}{ Name } & Betweenness & $\begin{array}{c}\text { Betweeness } \\
\text { centrality }\end{array}$ \\
\hline Gao Xiao-ping & 93.5 & 0.521 & Liu Jie & 33 & 0.184 \\
\hline XieYa-hong & 77 & 0.429 & Li Shan & 33 & 0.184 \\
\hline Wu Jian-nan & 71 & 0.395 & $\begin{array}{l}\text { Wang Ying- } \\
\text { luo }\end{array}$ & 17 & 0.095 \\
\hline Wen Hong & 56 & 0.312 & Da Qing-li & 5 & 0.028 \\
\hline He Zheng-fei & 45 & 0.251 & Hu Xian-zhi & 3.5 & 0.019 \\
\hline
\end{tabular}

Table 5 shows that: the majority of people are unable to keep the network connected in collaboration and the highest three are Gao Xiao-ping, Xie Ya-hong and $\mathrm{Wu}$ Jian-nan. Besides, the betweeness centrality of the 178 authors is zero except for the first 13 authors which proves that most of the authors who publish papers in the journals of management do not have the ability to control information exchange and participate less in academic communication. And that also proves the Two - Eight law in economics: Most of the resources are often monopolized by a small number of people. ${ }^{8}$

Table 6. Comparison of the top5 in Two Period

\begin{tabular}{|l|c|c|l|c|c|}
\hline & \multicolumn{2}{|c|}{$\mathbf{2 0 0 6 - 2 0 1 0}$} & & \multicolumn{2}{c|}{$\mathbf{2 0 1 1 - 2 0 1 5}$} \\
\hline \multicolumn{1}{|c|}{ Name } & Betweeness & $\begin{array}{c}\text { Betweeness } \\
\text { centrality }\end{array}$ & \multicolumn{1}{|c|}{ Name } & Betweeness & $\begin{array}{c}\text { Betweeness } \\
\text { centrality }\end{array}$ \\
\hline Jia Ling-ming & 2 & 0.014 & Li Shan & 10 & 0.092 \\
\hline Li Huan & 1 & 0.007 & Yang Shan-lin & 9 & 0.082 \\
\hline Liu Si-feng & 1 & 0.007 & Liu Jie & 6 & 0.055 \\
\hline Guo Ji & 1 & 0.007 & Gao Xiao-ping & 5 & 0.046 \\
\hline Ji Zhe1 & 0 & 0 & Wang Peng & 4 & 0.037 \\
\hline
\end{tabular}

Table 6 shows that in the latter five years, both the betweeness and betweeness centrality are increasing significantly, which indicates there are more authors involved in collaboration and their communication become more frequent.

\subsection{Core-Periphery Analysis}

The discussion of core-periphery has been hot for a long time, but rare of them are quantified by data. In the social network analysis, the nodes of the network center are the core authors in this filed. ${ }^{9}$ The purpose of this analysis are to identify the authors in the center and periphery.

The core-periphery structure of the author is analyzed by Ucinet. The first group is the core group, among which the Xia Shu-zhang is the core, and Ji Zhe, Gao Xiaoping are in the main core area. The second group is in periphery, the authors in this group collaborate less with others. This indicates that the members in group1 are capable of being a partner of others, while the group 2 members are always doing research by own self. This result is highly consistent with Fig.2. 
Paper-Analysis on the Co-authoring in the Field of Management in China: Based on Social Network...

Table 7. Core-Periphery Analysis Outcome

\begin{tabular}{|l|l|l|l|}
\hline \multicolumn{1}{|c|}{ 2006-2010 } & \multicolumn{1}{|c|}{ 2011-2015 } & \multicolumn{1}{c|}{ 2006-2010 } & \multicolumn{1}{c|}{ 2011-2015 } \\
\hline Xia Shu-zhang & Ji Zhe1 & Liu Jie & Chen Xiao-hong \\
\hline Ji Zhe1 & Xia Shu-zhang & He Jian-min & Ma Shi-hua \\
\hline Ji Zhe2 & Ji Zhe2 & She Hui & Hu Bin \\
\hline Liu Si-feng & Liu Si-feng & Shen Rong-hua & Zhao Shu-rong \\
\hline Tang Xiao-wo & Tang Xiao-wo & Zheng Cheng-wu & Zhang Qun \\
\hline Gao Xiaoping & Liu Si-feng & Zhang Zong-yi & Shi Ya-jun \\
\hline Liu Wei & Yang Shan-lin & Zhou Zhi-ren & Ye Fei \\
\hline Fang Zhi-geng & Gao Xiao-ping & Guo Ju-e & Zhou Jin \\
\hline Liang Liang & Zhang An-ding & Li Xiang-yang & Chen Tao \\
\hline Pu Yong-jian & He Jian-min & Hao Zheng-fei & Chen Hong \\
\hline$\ldots$ & $\ldots$ & $\ldots$ & $\ldots$ \\
\hline
\end{tabular}

Note: Jizhe1 and jizhe2 are homonyms of Chinese names.

Furthermore, Table 7 shows that the core members do not change in the two period and the core members are Xia Shu-zhang, Ji Zhe1, Ji Zhe2, Liu Si-feng, Tang Xiaowo, Gao Xiao-ping and so on. This reflects they are the authority in this field all the time. However, some authors changed their position and get into the core groups such as He Jian-min and Liu Jie.

\subsection{Small group analysis}

There are two methods for computing small groups: one is calculated by nodes and considered as a small group by the degree of nodes, and the other is calculated by distance and small groups at a certain distance.10 Small groups of research methods have many types, including factions, $n$-factions, $n$-sects, $k$-plex, $k$-core and so on. As the concept of $n$-factions is relatively close to the daily understanding of faction and the collaboration between authors does not have direction, we choose the undirected n-faction to calculate. 17 small groups are generated after the maximum distance $n$ between nodes and the number of small nodes are respectively set to 2 and 3. (Fig.3) 
Paper-Analysis on the Co-authoring in the Field of Management in China: Based on Social Network...

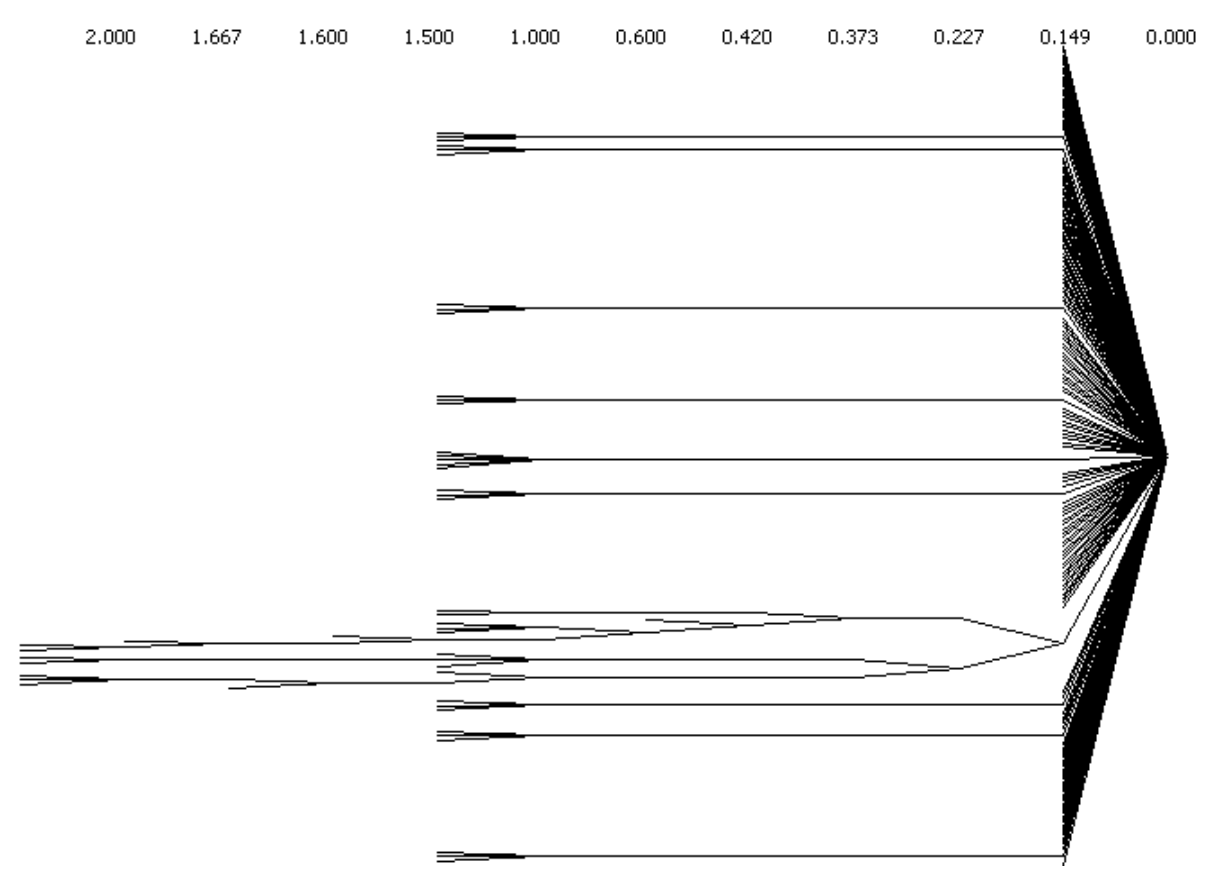

Fig. 3. $N$-cliques Analysis Outcome

As shown in Table 8 , there is one group with 8 members, three groups with five members, two groups with four members and eleven groups with three members. The small group shows some features:

1. The members in a group have a similar working experience. The largest coauthorship is based on Gao Xiao-ping, Liu Wei. Among them, Wu Jian-nan and Xie Ya-hong are also the important nodes, which play a role in establishing the connection with other authors. They are all the faculties of Chinese Institute of Administration and their research areas are public policy and administration.

2. Academic leaders play an important role in forming network. Taking Da Qing-li, Ji Jian-hua, Sun Hao, Wang Wen-ping and Wang Wen-bin for example. Daqing Li and Wang Wen-ping are famous professors from Southeast University and almost all of authors have relevant studying and working experience at Southeast University, which indicates that academic leaders can increase the possibility of scientific collaboration through their authority. As the core of the network, Da Qing-li is the key factor to maintain the link of the network, which reflects his important position in the field of management.

3. Geographicalcloseness is another vital factor to the establishment of networks. In the case of Liu Wei, Hu Xian-zhi and Jie Ya-hong. Liu Wei is an assistant professor in Beijing University of Chinese Medicine, Hu Xian-zhi is a Research Associate in Beihang University and Jie Ya-hong is the editor in Chinese Public Administration. The institutions of the authors are all located in Beijing. 
Table 8. Groups

\begin{tabular}{|c|c|c|c|}
\hline Group & member & Group & member \\
\hline 1 & $\begin{array}{l}\text { Gao Xiao-ping; Liu Wei; Shen Rong-hua; } \\
\text { Wang Lan-ming; Hu Xian-zhi; Jia Ling-min; } \\
\text { Wu Jian-nan; Xie Ya-hong }\end{array}$ & 10 & Lan Zhi-yong; Fan Zhi-ping; Liu Yang \\
\hline 2 & $\begin{array}{l}\text { Gao Xiao-ping; Liu Jie; Jia Ling-min; Wang } \\
\text { Ying-luo; Wu Jian-nan }\end{array}$ & 11 & Hao Hefei; Li Shan; Wen Hong \\
\hline 3 & $\begin{array}{l}\text { Liu Jie; Shang Hong; Wang Peng; Wu Jian- } \\
\text { nan }\end{array}$ & 12 & Hao He-fei;Xie Ya-hong; Wen Hong \\
\hline 4 & Wan Difang; Wang Ying-luo;Wu Jian-nan & 13 & $\begin{array}{l}\text { Guo Ju-e; Xi You-min; Zhuang Gui- } \\
\text { jun }\end{array}$ \\
\hline 5 & Ji Zhe; Hao He-fei; Li Shan;Zhang Hong-bin & 14 & $\begin{array}{l}\text { Chen Qi-an; Ren Yu-long; Yan Tai- } \\
\text { hua }\end{array}$ \\
\hline 6 & Liu Si-feng; Fang Geng-zhi; Zhu Jian-jun & 15 & $\begin{array}{l}\text { Gao Xiaoping; Hu Xian-zhi; Bao Jing; } \\
\text { Xie Ya-hong; Wen Hong }\end{array}$ \\
\hline 7 & $\begin{array}{l}\text { Da Qingli; Ji Jianhua; Sun Hao; Wang Wen- } \\
\text { ping; Wang Wen-bing }\end{array}$ & 16 & Fan Ying; Wei Yi-ming; Wu Gang \\
\hline 8 & Zhang An-ding; Yang Shan-lin;Zhang Qiang & 17 & Han Li-yan; Rao Yu-lei; Wang Ying \\
\hline 9 & Dan Bing; Zhang Xu-mei; Wang Lei & & \\
\hline
\end{tabular}

Table 9 shows that the number of collaboration groups has increased from 13 to 16 from the first five years to second. And the sizes of the groups have become larger. In the first five years, there are one group with five members, three groups with four members and nine groups with three members. And in the latter five years, there are four groups with six members, four groups with four members and eight groups with three members. It is worth noting that the groups are also in great changes. Some groups disappeared in the latter five years, such as group 4, 7, 9, 10, 11, 12 and13 while some groups come into being in the latter five years, such as group 1, 2, 3, 5, 8, $11,12,13,14,15$ and 16. Besides, the members in group change obviously, such as Chen Jian in group 5 in the former five years. Some members changed their group, such as Liu Jie and Liu Wei.

Table 9. Groups

\begin{tabular}{|c|c|c|c|}
\hline \multicolumn{2}{|r|}{ 2006-2010 } & \multicolumn{2}{|r|}{ 2011-2015 } \\
\hline Group & Author Name & Group & Author Name \\
\hline 1 & $\begin{array}{l}\text { Liu Jie; Gao Xiao-ping; Hu Xian-zhi; Jia } \\
\text { Ling-min }\end{array}$ & 1 & $\begin{array}{l}\text { Yang Shan-lin; Liang Chang-yong; Zhang } \\
\text { Qiang; Zhang Li; Luo He; Hu Xiao-xuan }\end{array}$ \\
\hline 2 & Liu Jie; Jia Ling-ming; Hao Zheng-fei & 2 & $\begin{array}{l}\text { Yang Shan-lin; Liang Chang-yong; } \\
\text { Jiang Bing }\end{array}$ \\
\hline 3 & $\begin{array}{l}\text { Gao Xiao-ping; Shen Rong-hua; Jia } \\
\text { Ling-min }\end{array}$ & 3 & $\begin{array}{l}\text { Yang Shan-lin; Luo He; Cao Jie; Hu Xiao- } \\
\text { xuan }\end{array}$ \\
\hline 4 & $\begin{array}{l}\text { Hu Xian-zhi; Jia Ling-min; Zhang Ji- } \\
\text { xing }\end{array}$ & 4 & $\begin{array}{l}\text { Liu Si-feng; Fang Zhi-geng; } \\
\text { Zhu Jian-jun }\end{array}$ \\
\hline 5 & $\begin{array}{l}\text { Liu Si-feng; Fang Zhi-geng; Chen Jian; } \\
\text { Zhu Jian-jun }\end{array}$ & 5 & $\begin{array}{l}\text { Ji Zhe; Zhang An-ding; Liu Jie; Li Shan; } \\
\text { Zheng Hao-fei; Shang Hong }\end{array}$ \\
\hline 6 & Liu Wei; Wu Gang; Li Chuan-zhao & 6 & $\begin{array}{l}\text { Gao Xiaoping; Liu Wei; Wang Lan-ming; } \\
\text { Shen Rong-hua; Wen Hong; Jie Ya-hong }\end{array}$ \\
\hline
\end{tabular}


Paper-Analysis on the Co-authoring in the Field of Management in China: Based on Social Network...

\begin{tabular}{|c|l|c|l|}
\hline 7 & Li Huan; Liu Yi; Gao Shan-xing & 7 & $\begin{array}{l}\text { Gao Xiao-ping; Liu Wei; Wang Lan-ming; } \\
\text { Shen Rong-hua; Jie Ya-hong; Bo Gui-li }\end{array}$ \\
\hline 8 & Gao Xiao-ping Guo Ji Shen Rong-hua & 8 & He Jian-min; Luo He; Cao Jie \\
\hline 9 & Guo Ji Shen Rong-hua Bao Jing & 9 & Liu Jie; Li Shan; Shang Hong; Wang Peng \\
\hline 10 & $\begin{array}{l}\text { Xiong Zhong-kai Zhao Quan-wu Bu } \\
\text { Xiang-zhi Chen Rong-qiu }\end{array}$ & 10 & Liu Jie; Wei Yu; Wang Peng \\
\hline 11 & Wu Chong-feng Li Jun Liu Hai-long & 11 & $\begin{array}{l}\text { Wang Shou-yang; Ma Chao-qun; Yang Xiao- } \\
\text { guang; Guo Ju-e }\end{array}$ \\
\hline 12 & $\begin{array}{l}\text { Li Li-ming Fan Ying Wu Gang Wei Yi- } \\
\text { ming Li Chuan-zhao }\end{array}$ & 12 & $\begin{array}{l}\text { Wang Shou-yang; Ma Chao-qun; Liu Wen- } \\
\text { bin; Zhou Zhong-bao }\end{array}$ \\
\hline 13 & Hu Xian-zhi Li Jun-qing Zhang Ji-xing & 13 & Da Qingli; SunHao; Hu Jin-song \\
\hline & & 14 & Li Shan; Hao Zheng-fei; WenHong \\
\hline & & 15 & Hao Zhengfei; Wen Hong; Jie Ya-hong \\
\hline & & 16 & Fan Zhi-ping; Lan Zhi-yong; Liuyang \\
\hline
\end{tabular}

\section{Conclusion}

Using bibliographic data extracted from CNKI database, social network analysis is used to generate and analyze the network of co-authors of China in the field of management. This article suggests that: the density of the network is low, which means the collaboration between authors in China is not tight; the relations between the degree centrality and research output are weak. The author who published more papers may not have more co-authors. Through the lens of betweenness centrality, several authors in key positions of network are always dominating the academic information exchange and the small groups of authors have changed from 2006 to 2015. The result of core-periphery analysis reflects that only a very small proportion of scholars are in the core of the network while most are relatively independent. The similarity of working experience, academic authority and geographical closeness are helpful to form and enhance the collaboration network.

\section{$7 \quad$ References}

[1] Cheong F, Corbitt B J. (2009) A social network analysis of the co-authorship network of the Australasian Conference of Information Systems from 1990 to 2006.European Conference on Information Systems, Ecis 2009, Verona, Italy. DBLP, 2009:292-303.

[2] Ozyar E, Gurdalli S. (2002) Social network analysis: a powerful strategy, also for the information sciences. Journal of Information Science (6):441-453.

[3] Qiu Jun-ping, Li Wei. (2012). An Empirical Analysis of Cooperative Patterns in Knowledge Transfer Area. Information studies: Theory \& Application (3).53-56

[4] Borgatti S P, Mehra A, Brass D J, et al.(2009) Network analysis in the social sciences. Science (5916), 892-895. https://doi.org/10.1126/science.1165821

[5] Krichel T, Bakkalbasi N. (2006). Social network analysis of research collaboration in the economics community. The International Workshop on Webometrics, Informetrics and Scientometrics \& Seventh COLLNET Meeting, Nancy France.

[6] Freeman, Linton C. (1997) A set of measures of centrality based on betweenness. Sociometry, Vol 40(1), Mar 1977, 35-41. https://doi.org/10.2307/3033543 
Paper-Analysis on the Co-authoring in the Field of Management in China: Based on Social Network...

[7] Abbasi A, Altmann J. (2011). On the Correlation between Research Performance and Social Network Analysis Measures Applied to Research Collaboration Networks. Hawaii International Conference on System Science. IEEE Xplore:1-10.

[8] Xu Jian, Huang Qiuyue. (2007). The Application of '2-8 Principles' in Library Management. Journal of Library Science In China, 33(5), 106-108.

[9] Jiang Chun-Lin, Zhang Fan, Tang Yue. (2010). Research on the Characteristics of Cocitation Network of Some Science Journals in China. Journal of Information, 29(4), 10-15.

[10] Scott J. (1988). Social Network Analysis. Sociology, 1988, 22(1):109-127. https://doi.org/10.1177/0038038588022001007

\section{Authors}

Chuanyi Wang is an associate professor in Institute of Educational Sciences Wuhan University, and Post-Doctoral Fellow of School of Information Management of Wuhan University. His research interests including Graduate Education, Ranking of University and etc.

Zhe Cheng (*Corresponding Author) is a master student in Institute of Educational Sciences Wuhan University.

Zhiwei Huang, corresponding author, is a master student in Institute of Educational Sciences Wuhan University.

Article submitted 01 May 2017. Published as resubmitted by the authors 10 June 2017. 